\title{
A Study on the Effects of Dry Needling in Multiple Sclerosis Patients with Spasticity: Protocol of a Randomized Waitlist-Controlled Trial
}

\author{
Omid Motamedzadeh ${ }^{1,2}$, Noureddin Nakhostin Ansari ${ }^{1,2,3, *}$, Soofia Naghdi ${ }^{1,2}$, Amirreza Azimi ${ }^{4}$, \\ Ashraf Mahmoudzadeh ${ }^{5}$, Sandra Calvo ${ }^{6}$, Pablo Herrero ${ }^{7}$ \\ ${ }^{1}$ Sports Medicine Research Center, Neuroscience Institute, Tehran University of Medical Sciences, Tehran, Iran \\ ${ }^{2}$ Department of Physiotherapy, School of Rehabilitation, Tehran University of Medical Sciences, Tehran, Iran \\ ${ }^{3}$ Research Center for War-affected People, Tehran University of Medical Sciences, Tehran, Iran \\ ${ }^{4}$ MS Research Center, Neurosciences Institute, Tehran University of Medical Sciences, Tehran, Iran \\ ${ }^{5}$ Musculoskeletal Research Center, Faculty of Rehabilitation Sciences, Isfahan University of Medical Sciences, Isfahan, Iran \\ 6iPhysio Research Group, Universidad San Jorge, Villanueva de Gállego, Zaragoza, Spain \\ ${ }^{7}$ Department of Physiatry and Nursing, Faculty of Health Sciences, University of Zaragoza, Zaragoza, Spain
}

Received July 20, 2020

Revised January 1, 2021

Accepted March 8, 2021

Correspondence to Noureddin Nakhostin Ansari Sports Medicine Research Center, Neuroscience Institute, Tehran University of Medical Sciences, Tehran, Iran

E-mail nakhostin@sina.tums.ac.ir
Background: Spasticity is a common symptom in multiple sclerosis (MS). Dry needling (DN) has been considered a useful method for the treatment of spasticity; however, there are no studies on the effects of DN on spasticity in patients with MS. We propose a study protocol aiming to investigate the effects of DN on spasticity in patients with MS.

Methods: MS patients with plantar flexor spasticity will be recruited. Participants will be randomly assigned to the DN group, where they will be receiving a single session of DN, one minute for each head of gastrocnemius muscle, or to the waiting list control group with no intervention. Primary outcome measures are the Modified Ashworth Scale (MAS) for gastrocnemius spasticity, passive resistive torque, and podography for foot pressure distribution. The ankle active and passive range of dorsiflexion and Timed Up and Go tests are the secondary outcome measures. All outcomes will be measured at baseline, immediately after the intervention, and one week later. A mixed-model, general linear model, and two-way repeated-measures ANOVA will be used to compare the quantitative variables between groups and within groups at the measurement time points. The MAS ordinal measure of spasticity will be compared between groups using the Kruskal-Wallis test, and both the Friedman test and Wilcoxon test will be used for within-group changes. Discussion: This study will provide primary evidence on the effects of DN on gastrocnemius muscle spasticity and gait in patients with MS.

Trial registration: Iranian Registry of Clinical Trials (IRCT): IRCT20190617043918N1.

Keywords: Multiple sclerosis, Dry needling, Spasticity, Gastrocnemius muscle

\section{INTRODUCTION}

Spasticity is one of the most common and debilitating symptoms associated with chronic neurological diseases, including multiple sclerosis (MS) [1-3]. In MS, muscle spasticity results from a release of proprioception reflexes in the spinal cord, which is due to an imbalance between the excitatory and inhibitory inputs to the alpha motor neuron [4]. The nature of the MS disease is dynamic and different among patients and still remains unpredictable [5]. The prevalence of spasticity in MS is high. A study found that $97 \%$ of patients with MS had lower limb spasticity and $50 \%$ of patients had upper limb spasticity [6]. Another study found that $84 \%$ of MS patients experienced a type of spasticity and $63 \%$ had problems with daily activities due to spasticity [7]. Severe spasticity, if not managed, can cause disability due to reduced mobility, pain, and disturbance in hygiene care. Consequently, spasticity is considered a significant burden for individuals with MS and their loved ones [8].

The therapeutic interventions used to manage spasticity 
include oral and injectable drug treatments, rehabilitation, and orthopedic or neurological surgery [9]. Medical treatments are expensive, have side effects, and are short-term and temporary [10-12]. Therefore, current interventions have limitations and complications and have not yielded satisfactory outcomes for treating MS-related spasticity $[13,14]$. It is, therefore, necessary to provide new and more effective interventions.

Dry needling (DN) is a treatment that has shown to be effective in decreasing spasticity in persons with stroke [15-19]. A systematic review summarized the evidence and concluded that DN had positive effects on spasticity, pain, and range of motion [20]. There are studies reported on the effects of DN on persons with cerebral palsy [21] and spinal cord injury [22, 23]. However, there are no studies on the effects of $\mathrm{DN}$ on the spasticity of patients with MS. The aim of this randomized waitlist-controlled clinical trial protocol is to investigate the effects of a single session of DN on plantar flexor spasticity immediately after treatment and one week after.

\section{MATERIALS AND METHODS}

\section{Study design}

This study will be a randomized waitlist-controlled clinical trial, based on the Standard Protocol Items: Recommendations for Interventional Trials (SPIRIT) guideline $[24,25]$, to investigate the effectiveness of DN on spastic gastrocnemius muscle in patients with MS. This study protocol has been independently peer-reviewed and approved by the Research Council of Sports Medicine Research Center, Neuroscience Institute, as well as the Research Council of School of Rehabilitation, Tehran University of Medical Sciences (TUMS), and approved by the Ethics Committee of Neuroscience Institute, TUMS (Reference number IR.TUMS. NI.REC.1399.004).

\section{Informed consent}

The study assessments and DN treatment will be described in detail to the participants. Before data collection, all individuals who agree to participate in this study will be asked to give their written informed consent. Subjects will be allowed to voluntarily withdraw from the study at any time without giving explanations or penalty. The physiotherapist who will assess the participants will also obtain the written informed consent.

\section{Study population}

Eligible individuals with MS will be recruited for this study in the period between July 2019 and December 2021.

\section{Inclusion and exclusion criteria}

Inclusion criteria will consist of patients with MS as diag- nosed by a neurologist, age $\geq 18$ years, no other concomitant neurodegenerative conditions, ability to walk with or without a supportive device, ability to understand commands, Expanded Disability Status Scale (EDSS) with a score of three to six, severity of spasticity of ankle plantar flexors being equal to or greater than one on the Modified Ashworth Scale (MAS). Exclusion criteria will consist of more than $10 \%$ limitation in the ankle passive range of motion (ROM), Botox injection three months prior to the current study, acute MS attack one month prior to the study initiation, taking antispasticity drugs, participation in any ongoing clinical trials, and presence of any kind of contraindication for $\mathrm{DN}$.

\section{Procedure}

The study will be performed at the Neurophysiotherapy Clinic, School of Rehabilitation, TUMS, Iran. Patients will be recruited from the Physical Therapy Clinic of the Iran MS Society and the MS Research Center, University Sina Hospital, Tehran, Iran. The eligible patients will be included based on the inclusion and exclusion criteria. Patients will be provided with oral and written information about the study. After reading and signing the consent form, patients will be randomized to either the DN or waiting list control group. The EDSS score will be determined by the neurologist who will be blinded to the groups and the assessment results. The entire outcomes will be randomly measured by a blinded assessor, an experienced and trained physiotherapist. The spasticity of plantar flexors will be evaluated using the MAS. The leg showing a higher level of spasticity will be selected. If the spasticity score of the two legs is equal, one of the legs will be randomly chosen for treatment. The recruitment of patients will begin in July 2019. We expect to end the data collection in December 2021. No person will have access to the trial dataset before the study's completion. Fig. 1 shows the schematic diagram of the study schedule of the procedure.

All study-related information will be stored securely at the study site. All participant information will be stored in locked file cabinets in areas with limited access. For data management, the data gathered from participants will be cleaned, coded, filed, and stored in numerical order in a password-secured PC. To ensure the accuracy of data entry, data will be double-checked to decrease the error rate and to identify missed or erroneous values.

\section{Randomization and blinding}

Eligible and willing patients referred by a neurologist will be randomly allocated to the $\mathrm{DN}$ or the waiting list control group. In order to randomly assign the patients, the strategy of opaque, sealed envelopes will be followed to ensure the concealment of 1:1 allocation, with two blocks of 12 patients using an online research randomizer sequence generator 


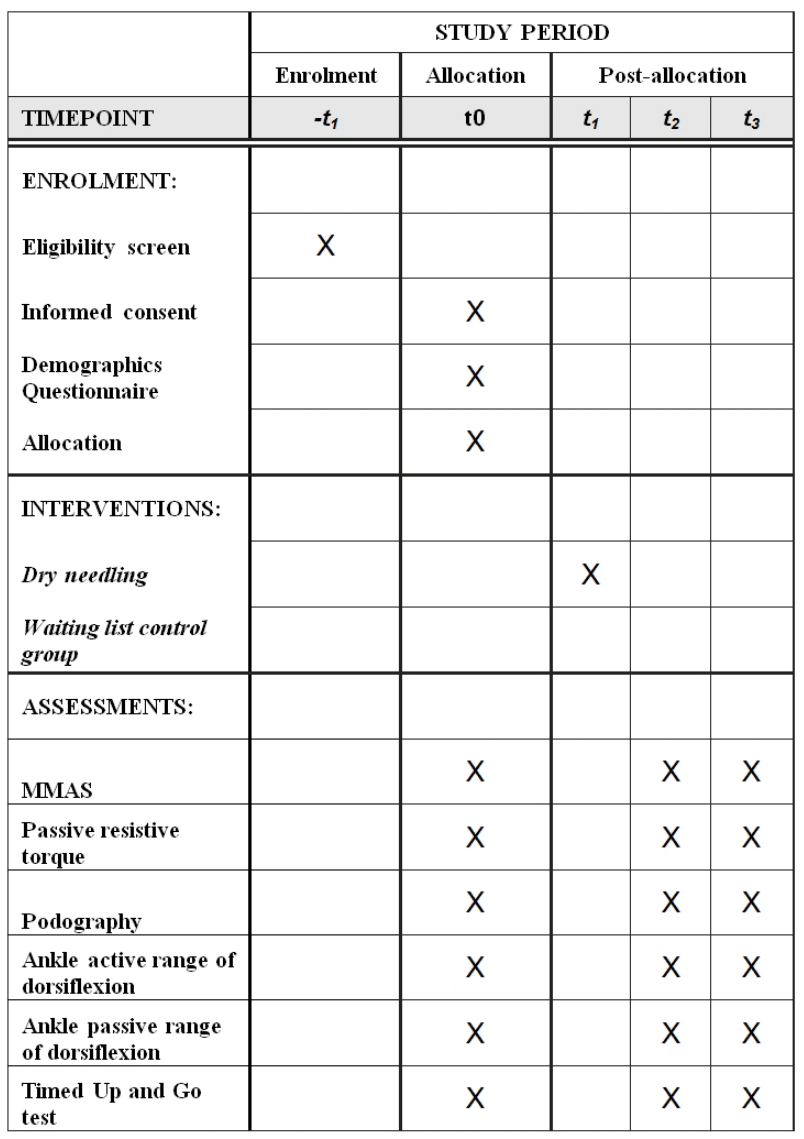

Note: SPIRIT, Standard Protocol Items: Recommendations for Interventional Trials; MMAS, Modified Modified Ashworth Scale; -t1: Pre-study, Screening/Consent; t0, Pre-study, Baseline/Randomization; t1, Study, Intervention; t2, Study, immediately after intervention; $\mathrm{t} 3$, one-week follow-up

Fig. 1. The SPIRIT schematic diagram of study schedule.

(http://www.randomizer.org). The outcomes assessor will be blinded to the assigned groups.

\section{Sample size}

We calculated the sample size for the current study protocol considering an effect size of 0.5 and the results of previous findings [16]. Assuming the alpha and power to be 0.05 and 0.8 , respectively, the total sample size needed is 24 (12 patients in each group). The neurologists and physiotherapists in the centers of the Iran MS Society and MS Research Center will continue screening patients for eligibility until the target population is achieved. To improve the adherence to the study protocol, increase the patients' retention in the study, and maximally complete the data collection, we adopted a short study duration strategy with a minimum treatment session (one session, two minutes of DN), with minimal time points for data collection between pre- and post-DN, and a short follow-up of one week. In the event of drop-outs, noncompliance, or missing outcomes, an intention-to-treat analysis will be executed.

\section{Intervention}

Subjects in the DN group will receive one session of DN for the gastrocnemius muscle, each head for one minute $[15,16]$. Subjects in the waiting list control group will not receive any intervention and will only be assessed. All the $\mathrm{DN}$ procedures will be provided by a licensed and experienced physiotherapist who has an MSc degree in physiotherapy with an official license to practice physiotherapy and use dry needling for treatment. The physiotherapist responsible for delivering the $\mathrm{DN}$ has seven years of experience in physiotherapy treating persons with various neurological conditions such as MS.

\section{Dry Needling (DN)}

$\mathrm{DN}$ is a method used by physiotherapists to treat myofascial trigger points and has been demonstrated to be effective in decreasing spasticity in patients with neurologic conditions [20]. Needles for the DN intervention will be disposable stainless steel sterilized needles $(0.25 \times 0.30$; DongBang AcuPrime Ltd, Korea). With the patient in the prone position with straight limbs and their feet hanging over the edge of a table, the fast-in and fast-out, cone shape technique $[15,16]$ will be applied for dry needling the gastrocnemius muscle. For the lateral head of the gastrocnemius muscle, a line from the middle of the heel to the middle of the popliteal fossa will be divided into three segments. The section $2 \mathrm{~cm}$ lateral to the middle of the third proximal segment will be needled. For the medial head of the gastrocnemius, the section $2 \mathrm{~cm}$ medial to the distal one third of proximal segment of the reference line will be needled. We will monitor and record any DNrelated adverse effects in the treatment session and after the conclusion of treatment at follow-up.

\section{Outcome measures}

The primary outcome measures will be the MAS scores for the plantar flexor muscles, passive resistive torque (PRT), and podography to determine the foot pressure distribution. An improvement in the range of active and passive ankle dorsiflexion and the Time Up and Go (TUG) test will be the secondary outcome measures. All outcome measures will be measured at baseline, immediately after the DN, and after one week.

\section{Spasticity}

The plantar flexor muscle spasticity will be evaluated by the MAS. First, the examiner will ask the patients to rest and relax without shoes on the bed for 5 minutes. For testing spasticity, the patient will be in the supine position with their knee joints in extension. The physiotherapist will stand on the affected side, and with one hand will stabilize the ankle joint, while holding the forefoot with his other hand. Then, the physiotherapist will move the ankle joint from the 
maximum possible plantar flexion to the maximal possible dorsiflexion by counting "one thousand one" to stretch the spastic muscles over the duration of about one second [26]. The passive movement will be performed only one time. The physiotherapist will determine the intensity of spasticity based on the MAS scale and the resistance felt during the passive movement. The MAS is scored on an ordinal scale from zero to four (Table 1). The reliability and validity of MAS for lower limb muscle spasticity have been demonstrated [27]. In this study, the Persian version of MAS will be used [28].

\section{Passive resistive torque}

The PRT will be assessed using a manual micro-muscle tester (microFET ${ }^{\circledR} 3$, HOGGAN Scientific, USA). The procedure used to score the spasticity of the gastrocnemius will be followed for passive torque (N.m) measurement except that the movements will be made through the dynamometer [29]. Studies have demonstrated the reliability and validity of the manual dynamometer for evaluating the passive plantarflexion torque in spastic patients [30,31].

\section{Foot pressure}

A capacitance-based pressure platform $\left(\right.$ Emed $^{\circledR} \mathrm{M}$ pressure platform) will be used for detecting the foot pressure distribution. The pressure platform is $380 \times 240 \mathrm{~mm}$ and resolution of four sensors per $\mathrm{cm}^{2}$ (sensor area, $475 \times 320 \mathrm{~mm}$ ) when data are collected at $50 \mathrm{~Hz}$. All the sensors of the platforms will operate with calibration.

To collect the data, the participants will be asked to walk barefoot across the platform at a preferred and comfortable walking speed. The participants will be asked to focus on a rounded sticker fixed in both directions at the same level from the ground during the measurement. A long walkway will be used so participants will be required to take four steps prior to hitting the platform and continuing to walk afterward. These procedures will be repeated until five passes

Table 1. The Modified Ashworth Scale [28]

\begin{tabular}{|c|c|}
\hline Grade & Definition \\
\hline 0 & No increase in muscle tone \\
\hline 1 & $\begin{array}{l}\text { Slight increase in muscle tone, manifested by a catch and } \\
\text { release or by minimal resistance at the end of the range of } \\
\text { motion when the affected part(s) is moved in flexion/ } \\
\text { extension }\end{array}$ \\
\hline 2 & $\begin{array}{l}\text { Marked increase in muscle tone, manifested by a catch in } \\
\text { the middle range and resistance throughout the remainder } \\
\text { of the range of motion, but the affected part(s) is easily } \\
\text { moved }\end{array}$ \\
\hline 3 & $\begin{array}{l}\text { Considerable increase in muscle tone, passive movement is } \\
\text { difficult }\end{array}$ \\
\hline 4 & Affected part(s) is rigid in flexion or extension \\
\hline
\end{tabular}

are obtained (five recordings of the affected foot), then an average of the five steps on the affected foot will be used to represent the individual's dynamic foot placement. The trial will be repeated if the foot is placed near to or on the edges of the platform. To analyze force and pressure, the foot will be divided into 10 regions: hindfoot, midfoot, first metatarsal, second metatarsal, third metatarsal, fourth metatarsal, fifth metatarsal, big toe, second toe, and toes three, four, and five. The contact area $\left(\mathrm{cm}^{2}\right)$, maximum force $(\mathrm{N})$, and peak pressure (Kpa) will be calculated for the analyses. The reliability of the measurements has been shown in previous studies [32].

\section{Ankle dorsiflexion}

The passive and active ROM of ankle dorsiflexion will be measured with an ankle biplane goniometer (A Bissell Health Care, model 7524, USA) as used previously [33]. The measurements will be performed with the patient in the supine position and the knee extended to primarily measure the gastrocnemius spasticity [29]. The maximal passive ROM (PROM) will be evaluated while the physiotherapist passively moves the ankle joint to its maximal dorsiflexion. The active ROM (AROM) will be measured while the patient voluntarily dorsiflexes the ankle joint [34]. The biplane goniometer has a plantar platform as the movable arm to maximize the control of the entire foot when moving it.

\section{Functional mobility}

The functional mobility of patients will be assessed using the TUG test of walking. It is a simple test of walking speed requiring both static and dynamic balance as it involves standing from the sitting position, walking for three meters, turning, and sitting down again. The reliability and validity of the TUG test have been demonstrated in neurological diseases, including MS [35-38].

\section{Data monitoring}

The study procedure will be monitored by an independent committee from various disciplines to ensure adherence to the method and accuracy of the accumulated data.

\section{Data collection and analysis}

Data from participants who completed the study protocol will be included for statistical analyses. The KolmogorovSmirnov test will be used to determine the normal distribution of the data. For data that are normally distributed, the mean \pm SD will be calculated; otherwise, the median (interquartile range) will be reported. Differences between groups on the demographic data will be analyzed with independent samples t-test or Mann-Whitney U test. The two-way Repeated Measures ANOVA will be used 
for continuous outcome measures with the groups as the between-subject factor (2 groups) and one within-subject factor (three measurements at before, immediately after, and at follow-up). Pairwise comparisons between the measurement time points will be analyzed by Bonferroni corrections. The Greenhouse-Geisser estimates of sphericity will be used to correct the degrees of freedom if the homogeneity of variances, calculated by Mauchly's test, were not met. For the ordinal measure of MAS, the Friedman's test will be used with post hoc Wilcoxon signed-rank test (WSRT) for paired comparisons; the Kruskal-Wallis test will be used for between-group comparisons. The size of the treatment effect will be examined for groups with Cohen's $d$. We will use SPSS version 18 (SPSS, Chicago; IL) with alpha at $\leq 0.05$ significance.

\section{DISCUSSION}

In this study, we first aimed to determine whether DN has a significant effect on spasticity in patients with MS compared to the waiting list control group as shown in previous reports in stroke $[15-19,39]$. The second purpose is to determine whether DN can improve foot pressure distribution, PRT, and ROM in response to possible improvements in spasticity based on MAS scores. Finally, we will examine whether DN has a significant effect on functional walking. To our knowledge, no study has reported the effectiveness of DN in MS-related spasticity and gait.

Spasticity is common in MS. There is currently a wide range of preventive, medical, and rehabilitation techniques to manage spasticity. However, the application of interventions depends on the etiology of spasticity. It is known that the features of spasticity have differences between MS and stroke [40]. Although there are positive effects of $\mathrm{DN}$ in spasticity reduction after stroke [15-18,39], no reports exist on the effectiveness of DN on spasticity in patients with MS.

Although the reason for spasticity is neural in origin, muscle structural changes occur as a consequence of spasticity [41]. It follows that the spasticity together with mechanical characteristics of spastic muscle contributes to the increase of PRT, limitations in active and passive ROM, and functional disability. We expect immediate reductions in the spasticity of the gastrocnemius muscle after DN, as it is known that DN provokes a mechanical disruption of dysfunctional motor endplates and neurophysiological effects (e.g., improvement in motor neuron excitability, activating the neural inhibitory system) $[15,16]$.

Muscle spasticity following upper motor neuron dysfunction, together with mechanical alterations, can cause muscle stiffness appearing clinically as increased PRT. Reduction in spasticity post-DN may contribute to the sub- sequent improvements in PRT. A study in patients with stroke found a significant correlation between the passive resistance force and the passive ROM in patients with poststroke spasticity [16]. Hence, improvements in both spasticity and PRT through changes in neural and muscle mechanical properties have led us to hypothesize improvements in passive ROM post-DN.

We expect improvement in the ankle active ROM after DN as spasticity influences active movements. Although impairments in active voluntary movements result primarily from impairment in central commands in recruiting motor neurons, a study in patients with stroke found a negative correlation between the active ROM and the spasticity MAS scores (Spearman's rho $=-0.42$ ) and a direct correlation between the active ROM and the passive ROM ( $r=0.49)$ [16]. We hypothesize that with reductions in spasticity and PRT as well as improvements in ankle passive ROM, improvements in the ankle active ROM will be demonstrated after DN in patients with MS recruited for this study.

The podography platforms are used in this study to collect data for assessing plantar pressure distribution and analyzing foot function. Improving plantar pressures in the feet is an important step for improving the walking ability in patients with MS. We expect to find significant changes in pressure distribution, such that patients receiving DN will experience an increase in contact area compared to those in the control group [18]. Decreases in the gastrocnemius spasticity and PRT would explain the improvements in contact surface after DN. The increases in the contact area of the foot after DN will, in turn, decrease the abnormal localized peak plantar pressures leading to improved walking ability.

We considered the TUG test as a functional measure to quantify gait improvements and that any reductions in gastrocnemius spasticity and/or improvements in plantar pressure distribution will improve the gait performance. It will be evaluated if there are changes in gait and if these changes are clinically meaningful, as well as if they are related to any of the other changes in the rest of the variables.

This study is the first randomized waitlist-controlled trial to use a single session of DN to treat gastrocnemius spasticity in individuals with MS. Our study findings will analyze if the positive effects of $\mathrm{DN}$ reported in previous studies of spasticity post-stroke will extend to other neurological conditions such as MS. The single DN session without repeated task-oriented exercises of sufficient doses may limit the clinically relevant changes in outcomes. The major limitation is that the longterm effects will not be evaluated.

\section{FUNDING}

This study project is financially supported by a grant from 
Sports Medicine Research Center, Neuroscience Institute, Tehran University of Medical Sciences (grant number: 98-0253-43385). The funder will only support the study with fund with no role in the study design or in writing the manuscript. The results obtained from this study will be disseminated in a peer-reviewed journal and presented at national/international congresses.

\section{ACKNOWLEDGEMENTS}

We wish to thank the Sports Medicine Research Center, Neuroscience Institute, Tehran University of Medical Sciences for supporting this study. We also appreciate the Research Deputy, Tehran University of Medical Sciences.

\section{AUTHORS' CONTRIBUTIONS}

NNA, SN, and OM presented the study conception and idea. NNA, SN, OM, AA, AM, SC, and PHG designed the study protocol. OM drafted the first version of this manuscript that was reviewed and revised critically for intellectual content by NNA. Revisions were performed by NNA, SN, OM, SC, and PH. All authors read, commented, and approved the final manuscript for submission.

\section{CONFLICT OF INTEREST}

The authors declare no conflict of interest.

\section{ORCID}

Omid Motamedzadeh, https://orcid.org/0000-0002-5149-5651

Noureddin Nakhostin Ansari, https://orcid.org/0000-0003-2742-2273

Soofia Naghdi, https://orcid.org/0000-0002-5062-7747

Amirreza Azimi, https://orcid.org/0000-0002-0966-7114 Ashraf Mahmoudzadeh, https://orcid.org/0000-0002-1416-2102

Sandra Calvo, https://orcid.org/0000-0002-1674-7788

Pablo Herrero, https://orcid.org/0000-0002-9201-0120

\section{REFERENCES}

1. Arroyo R, Massana M, Vila C. Correlation between spasticity and quality of life in patients with multiple sclerosis: the CANDLE study. Int J Neurosci 2013;123:850-8.

2. Eyssette M, Rohmer F, Serratrice G, Warter JM, Boisson D. Multi-centre, double-blind trial of a novel antispastic agent, tizanidine, in spasticity associated with multiple sclerosis. Curr Med Res Opin 1988;10:699-708.
3. Haas J. Pathophysiology, assessment and management of multiple sclerosis spasticity: an update. Expert Rev Neurother 2011;11(4 Suppl):3-8.

4. Zhang LQ, Chen K, Kang SH, Sliwa JA, Cohen BA, Rymer WZ, et al. Characterizations of reflex and nonreflex changes in spastic multiple sclerosis. J Neurosci Methods 2014;231:3-8.

5. Patejdl R, Zettl UK. Spasticity in multiple sclerosis: contribution of inflammation, autoimmune mediated neuronal damage and therapeutic interventions. Autoimmun Rev 2017;16:925-36.

6. Barnes MP, Kent RM, Semlyen JK, McMullen KM. Spasticity in multiple sclerosis. Neurorehabil Neural Repair 2003;17:66-70.

7. Rizzo MA, Hadjimichael OC, Preiningerova J, Vollmer TL. Prevalence and treatment of spasticity reported by multiple sclerosis patients. Mult Scler 2004;10:589-95.

8. Milinis K, Young CA; Trajectories of Outcome in Neurological Conditions (TONiC) study. Systematic review of the influence of spasticity on quality of life in adults with chronic neurological conditions. Disabil Rehabil 2016;38:1431-41.

9. Chang E, Ghosh N, Yanni D, Lee S, Alexandru D, Mozaffar T. A review of spasticity treatments: pharmacological and interventional approaches. Crit Rev Phys Rehabil Med 2013;25:11-22.

10. Bethoux F. Spasticity management after stroke. Phys Med Rehabil Clin N Am 2015;26:625-39.

11. Graham LA. Management of spasticity revisited. Age Ageing 2013;42:435-41.

12. Kaku M, Simpson DM. Spotlight on botulinum toxin and its potential in the treatment of stroke-related spasticity. Drug Des Devel Ther 2016;10:1085-99.

13. Sommerfeld DK, Gripenstedt U, Welmer AK. Spasticity after stroke: an overview of prevalence, test instruments, and treatments. Am J Phys Med Rehabil 2012;91:814-20.

14. Rekand T. Clinical assessment and management of spasticity: a review. Acta Neurol Scand Suppl 2010;(190):62-6.

15. Ansari NN, Naghdi S, Fakhari Z, Radinmehr H, Hasson S. Dry needling for the treatment of poststroke muscle spasticity: a prospective case report. NeuroRehabilitation 2015;36:61-5.

16. Fakhari Z, Ansari NN, Naghdi S, Mansouri K, Radinmehr H. A single group, pretest-posttest clinical trial for the effects of dry needling on wrist flexors spasticity after stroke. NeuroRehabilitation 2017;40:325-36.

17. Calvo S, Quintero I, Herrero P. Effects of dry needling (DNHS technique) on the contractile properties of spastic muscles in a patient with stroke: a case report. Int J Rehabil Res 2016;39:372-6.

18. Salom-Moreno J, Sánchez-Mila Z, Ortega-Santiago R, PalaciosCeña M, Truyol-Domínguez S, Fernández-de-las-Peñas C. Changes in spasticity, widespread pressure pain sensitivity, and baropodometry after the application of dry needling in patients who have had a stroke: a randomized controlled trial. J Manipulative Physiol Ther 2014;37:569-79.

19. Mendigutia-Gómez A, Martín-Hernández C, Salom-Moreno J, Fernández-de-Las-Peñas C. Effect of dry needling on spasticity, 
shoulder range of motion, and pressure pain sensitivity in patients with stroke: a crossover study. J Manipulative Physiol Ther 2016;39:348-58.

20. Valencia-Chulián R, Heredia-Rizo AM, Moral-Munoz JA, Lucena-Anton D, Luque-Moreno C. Dry needling for the management of spasticity, pain, and range of movement in adults after stroke: a systematic review. Complement Ther Med 2020;52:102515.

21. Herrero Gallego P, Mayoral del Moral O. A case study looking at the effectiveness of deep dry needling for the management of hypertonia. J Musculoskelet Pain 2007;15:55-60.

22. Fresno M, Mediavilla P, Mayoral O. Dry needling of myofascial trigger points for hypertonia spastica in incomplete spinal cord injuries: report of two cases. J Musculoskelet Pain 2004;12:75.

23. Cruz-Montecinos C, Núñez-Cortés R, Bruna-Melo T, Tapia C, Becerra P, Pavez N, et al. Dry needling technique decreases spasticity and improves general functioning in incomplete spinal cord injury: a case report. J Spinal Cord Med 2020;43:414-8.

24. Chan AW, Tetzlaff JM, Altman DG, Laupacis A, Gøtzsche PC, Krleža-Jerić K, et al. SPIRIT 2013 statement: defining standard protocol items for clinical trials. Ann Intern Med 2013;158:200-7.

25. Chan AW, Tetzlaff JM, Gøtzsche PC, Altman DG, Mann H, Berlin JA, et al. SPIRIT 2013 explanation and elaboration: guidance for protocols of clinical trials. BMJ 2013;346:e7586.

26. Ansari NN, Naghdi S, Moammeri H, Jalaie S. Ashworth Scales are unreliable for the assessment of muscle spasticity. Physiother Theory Pract 2006;22:119-25.

27. Ghotbi N, Nakhostin Ansari N, Naghdi S, Hasson S. Measurement of lower-limb muscle spasticity: intrarater reliability of Modified Modified Ashworth Scale. J Rehabil Res Dev 2011;48: 83-8.

28. Nakhostin Ansari N, Naghdi S, Forogh B, Hasson S, Atashband M, Lashgari E. Development of the Persian version of the Modified Modified Ashworth Scale: translation, adaptation, and examination of interrater and intrarater reliability in patients with poststroke elbow flexor spasticity. Disabil Rehabil 2012;34:1843-7.

29. Radinmehr H, Nakhostin Ansari N, Naghdi S, Olyaei G, Tabatabaei A. Effects of one session radial extracorporeal shockwave therapy on post-stroke plantarflexor spasticity: a single-blind clinical trial. Disabil Rehabil 2017;39:483-90.

30. Lamontagne A, Malouin F, Richards CL, Dumas F. Evaluation of reflex- and nonreflex-induced muscle resistance to stretch in adults with spinal cord injury using hand-held and isokinetic dynamometry. Phys Ther 1998;78:964-75; discussion 976-8.

31. Lorentzen J, Grey MJ, Geertsen SS, Biering-Sørensen F, Brunton $\mathrm{K}$, Gorassini M, et al. Assessment of a portable device for the quantitative measurement of ankle joint stiffness in spastic individuals. Clin Neurophysiol 2012;123:1371-82.

32. Putti AB, Arnold GP, Cochrane LA, Abboud RJ. Normal pressure values and repeatability of the Emed ST4 system. Gait Posture 2008;27:501-5.

33. Ansari NN, Naghdi S, Bagheri H, Ghassabi H. Therapeutic ultrasound in the treatment of ankle plantarflexor spasticity in a unilateral stroke population: a randomized, single-blind, placebo-controlled trial. Electromyogr Clin Neurophysiol 2007; 47:137-43.

34. Nakhostin Ansari N, Naghdi S, Hasson S, Rastgoo M. Efficacy of therapeutic ultrasound and infrared in the management of muscle spasticity. Brain Inj 2009;23:632-8.

35. Nilsagard Y, Lundholm C, Gunnarsson LG, Dcnison E. Clinical relevance using timed walk tests and 'timed up and go' testing in persons with multiple sclerosis. Physiother Res Int 2007;12:10514.

36. Ng SS, Hui-Chan CW. The timed up \& go test: its reliability and association with lower-limb impairments and locomotor capacities in people with chronic stroke. Arch Phys Med Rehabil 2005;86:1641-7.

37. Morris S, Morris ME, Iansek R. Reliability of measurements obtained with the Timed "Up \& Go" test in people with Parkinson disease. Phys Ther 2001;81:810-8.

38. Nordin E, Rosendahl E, Lundin-Olsson L. Timed "Up \& Go" test: reliability in older people dependent in activities of daily living--focus on cognitive state. Phys Ther 2006;86:646-55.

39. Sánchez-Mila Z, Salom-Moreno J, Fernández-de-Las-Peñas C. Effects of dry needling on post-stroke spasticity, motor function and stability limits: a randomised clinical trial. Acupunct Med 2018;36:358-66.

40. Picelli A, Vallies G, Chemello E, Castellazzi P, Brugnera A, Gandolfi M, et al. Is spasticity always the same? An observational study comparing the features of spastic equinus foot in patients with chronic stroke and multiple sclerosis. J Neurol Sci 2017;380:132-6.

41. Lieber RL, Steinman S, Barash IA, Chambers H. Structural and functional changes in spastic skeletal muscle. Muscle Nerve 2004;29:615-27. 